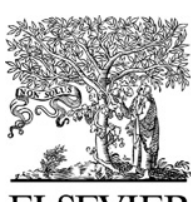

\section{Treatment of advanced chronic heart failure with normal left ventricular ejection fraction. Response to the letter by Dr. Martinez-Selles}

We thank Dr. Martinez-Selles for his letter regarding our position statement on advanced chronic heart failure (ACHF). As he correctly points out, in our definition of ACHF we have included patients with normal left ventricular ejection fraction (LVEF). To increase the specificity of our diagnostic criteria, we allowed inclusion of these patients only if they had other signs of severe cardiac dysfunction (i.e. a restrictive or pseudonormal mitral inflow pattern at Doppler-echocardiography and/or high left or right ventricular filling pressure at right heart catheterisation and/or high BNP or NT-ProBNP plasma levels).[1]

Dr. Martinez-Selles notes that we did not differentiate between the treatment of the patients with normal LVEF and that of the patients with low LVEF. With respect to medical treatment, we recognise in accordance with the current guidelines, [2] that there is little evidence in favour of any specific drug regimen. However, based on the mechanisms leading to symptom and disease progression in patients with heart failure and normal LVEF and on several clinical trials recently accomplished, we concluded that there are no major differences in the medical treatment of the patients with a normal LVEF, as compared to those with a reduced LVEF. This is also the position taken in the current European Society of Cardiology guidelines. [2] We have, therefore, not discussed medical treatment of ACHF patients with low or normal LVEF separately. This would be an appropriate target for further research.

On the other hand, with respect to the indications for devices and again in agreement with current guidelines, we do not think devices should be implanted in patients with heart failure and normal LVEF.[2] There is very limited evidence collected in this population. In our position statement we refer to the current guidelines and recommendations for an implantable cardioverter defibrillator (ICD) and/or cardiac resynchronisation therapy (CRT). With respect to CRT, we wrote that it has been shown to reduce mortality and hospitalisations and improve symptoms and quality of life in patients with reduced LVEF and wide QRS duration. It would be logical to consider patients with these characteristics and ACHF as candidates [1].

In conclusion, we agree with Dr. Martinez-Selles with regard of the importance of ACHF with preserved LVEF.
However, we do not believe that there are major differences in pharmacological treatment. However, the indications for ICD or CRT should currently be restricted to patients with demonstrable systolic dysfunction.

\section{References}

[1] Metra M, Ponikowski P, Dickstein K, et al. Advanced chronic heart failure: a position statement from the Study Group on Advanced Heart Failure of the Heart Failure Association of the European Society of Cardiology. Eur J Heart Fail 2007;9:684-94.

[2] Swedberg K, Cleland J, Dargie H, et al. Guidelines for the diagnosis and treatment of chronic heart failure: executive summary (update 2005): the task force for the diagnosis and treatment of chronic heart failure of the European Society of Cardiology. ESC Committee for Practice Guidelines. Eur Heart J 2005;26:1115-40.

Marco Metra

Section of Cardiovascular Diseases, Department of Experimental and Applied Medicine, University of Brescia, Italy Corresponding author.

Section of Cardiovascular Diseases, c/o Cardiologia, Spedali Civili, P.zza Spedali Civili, 25123 Brescia, Italy. Tel.: +39 30 3995572; fax: +39 303700359 . E-mail address: metramarco@libero.it.

Dirk Brutsaert

Department of Cardiology, A.Z. Middellheim Hospital, Univ. of Antwerp, Antwerp, Belgium

Kenneth Dickstein Cardiology Division, University of Bergen, Stavanger University Hospital, Stavanger, Norway

Alan G. Fraser Department of Cardiology, Wales Heart Research Institute, University of Wales College of Medicine, Cardiff, UK

$$
\begin{array}{r}
\text { Claes-Hakan Bergh } \\
\text { Department of Cardiology, } \\
\text { Sahlgrenska University Hospital/Sahlgrenska, } \\
\text { Göteborg, Sweden }
\end{array}
$$

Michael Böhm Innere Medizin III, Universitätskliniken des Saarlandes, Homburg/Saar, Germany 
Antonello Gavazzi Department of Cardiology, Ospedali Riuniti di Bergamo, Bergamo, Italy

Tiny Jaarsma Department of Cardiology, Programme Coördinator $\mathrm{COACH}$, University Hospital Groningen, Groningen, The Netherlands
Michel Komajda Département de Cardiologie, Pitié Salpêtrière Hospital, Paris Cedex 13, France

On behalf of the Study Group on Advanced Heart Failure of the Heart Failure Association of the European Society of Cardiology

26 August 2007

Piotr Ponikowski Department of Cardiology, Military Hospital,

Wroclaw, Poland 